\title{
Magnetic properties of thin Ni films measured by a dc SQUID-based magnetic microscope
}

Snigirev, O.V.; Andreev, K.E.; Tishin, A.M.; Gudoshnikov, S.A.; Bohr, Jakob

Published in:

Physical Review B

Link to article, DOI:

10.1103/PhysRevB.55.14429

Publication date:

1997

Document Version

Publisher's PDF, also known as Version of record

Link back to DTU Orbit

Citation (APA):

Snigirev, O. V., Andreev, K. E., Tishin, A. M., Gudoshnikov, S. A., \& Bohr, J. (1997). Magnetic properties of thin Ni films measured by a dc SQUID-based magnetic microscope. Physical Review B, 55(21), 14429-14433. https://doi.org/10.1103/PhysRevB.55.14429

\section{General rights}

Copyright and moral rights for the publications made accessible in the public portal are retained by the authors and/or other copyright owners and it is a condition of accessing publications that users recognise and abide by the legal requirements associated with these rights.

- Users may download and print one copy of any publication from the public portal for the purpose of private study or research.

- You may not further distribute the material or use it for any profit-making activity or commercial gain

- You may freely distribute the URL identifying the publication in the public portal 


\title{
Magnetic properties of thin Ni films measured by a dc SQUID-based magnetic microscope
}

\author{
O. V. Snigirev, K. E. Andreev, and A. M. Tishin \\ Faculty of Physics, M.V. Lomonosov Moscow State University, Moscow, 119899, Russia \\ S. A. Gudoshnikov \\ Institute of Terrestrial Magnetism, Ionosphere and Radio Wave Propagation, Russian Academy of Sciences, 142092, Moscow region, \\ Troitsk, Russia \\ J. Bohr \\ Department of Physics, Technical University of Denmark, Building 307, DK-2800 Lyngby, Denmark
}

(Received 21 October 1996)

\begin{abstract}
We have applied a scanning HTS (high-temperature superconductor) dc SQUID (superconducting quantum interference device) -based magnetic microscope to study the magnetic properties of $\mathrm{Au} / \mathrm{Ni} / \mathrm{Si}(100)$ films in the thickness range from 8 to $200 \AA$ at $T=77 \mathrm{~K}$. A one-domain structure with in-plane orientation of the magnetic moment was found for film thicknesses exceeding $26 \AA$. A drastic decrease of the magnetization of the film was detected when the thickness is less than $26 \AA$. [S0163-1829(97)08821-8]
\end{abstract}

Magnetism of ultrathin magnetic structures is of both practical and fundamental interest. Thin films with perpendicular magnetization can be used in magnetic recording devices with high storage density. From the fundamental viewpoint, thin films are interesting for investigating magnetic ordering in two-dimensional (2D) magnets. ${ }^{1,2}$ The properties of magnetic films depends on the film thickness. The Curie temperature $T_{C}$, the value of the magnetization $M$, the orientation of the magnetic moment, etc. can depend drastically on the thickness and structure of the film, and on the method of film preparation. Measurements of the magnetization of thin magnetic films or even monolayers (ML) require highly sensitive experimental techniques due to the small quantity of magnetic atoms under investigation.

Typically one of the following three types of devices are used for measuring the magnetic properties of thin films, surfaces, and submicron particles.

(A) Devices capable of measuring an integral magnetic moment such as a vibrating sample magnetometer, ${ }^{3}$ a torsion oscillation magnetometer, ${ }^{4}$ and a cantilever beam technique. ${ }^{5}$

(B) Devices which provide high-resolution imaging of magnetic structures such as a magnetic force microscope, and magneto-optic Kerr effect device. ${ }^{6-8}$

(C) Synchrotron source photon beams techniques. The microscopic magnetism can be studied by this method ${ }^{9,10}$ and resonant photon scattering can be used in connection with studies of the surface magnetism. ${ }^{10,11}$

A recently invented scanning superconducting quantum interference device (SQUID) microscope (SSM) (see, for example, Ref. 12) provides an example of a very attractive technique for these types of measurements. With respect to the other mentioned devices, the SSM enables the magnetization to be determined with high sensitivity and images to be recorded of the magnetic structure with space resolution down to a few micrometers. ${ }^{13}$ In the present work we have developed an experimental HTS (high-temperature superconductor) dc SQUID technique capable of investigating the local magnetization of ultrathin films. In this paper we report on the magnetic properties of ultrathin $\mathrm{Au} / \mathrm{Ni} / \mathrm{Si}(100)$ films at $77 \mathrm{~K}$ as a function of the thickness of the Ni layers.

The Ni films are characterized by specific magnetic properties. According to a theoretical prediction, fcc Ni exists only in a ferromagnetic phase, while bcc Ni exhibits a transition from a ferromagnetic to a nonmagnetic phase, ${ }^{14}$ when the Wigner-Seitz radius $r_{\mathrm{WS}}$ decreases to a critical value of about $2.60 \AA$. The transition from a nonmagnetic to ferromagnetic state has been predicted for epitaxial bcc Ni films which are subject to a $1 \%$ lattice expansion caused by a substrate. $^{14}$

The magnetic moments in Ni films exhibit a strong dependence on the number of monolayers, the substrate, and the structure of the covering (cap) layers. For example, the magnetic moment of bcc Ni film deposited on a Fe(001) substrate is $0.86 \mu_{B}$ per atom for a one monolayer thick film, and $0.69 \mu_{B}$ for a two monolayer thick film. ${ }^{15}$ It has been suggested that the magnetic moment decreases only in the first $\mathrm{Ni}$ layer of a single crystal covered by $\mathrm{Cu}$ layers. ${ }^{16}$ Nevertheless, a large discrepancy still exist between the experimental data ${ }^{17}$ and the theoretical estimates of the magnetic moment in the few monolayer limit. ${ }^{14,15}$

It has been observed that the orientation of magnetization in epitaxial Ni films depends on the film thickness. ${ }^{7,18}$ For thicknesses less than 7 layers the magnetization is in-plane, while the easy direction is out of plane for thicker films. It has been demonstrated that such types of systems can exhibit a perpendicular magnetization in a wide range of thicknesses [10-60 $\AA$ of $\mathrm{Ni}$ for vacuum $/ \mathrm{Ni} / \mathrm{Cu} / \mathrm{Si}(001)$ and $20-135 \AA$ of $\mathrm{Ni}$ for $\mathrm{Cu} / \mathrm{Ni} / \mathrm{Cu} / \mathrm{Si}(001)]^{6,8}$ Various domain sizes have been observed $^{6}$ in $\mathrm{Ni} / \mathrm{Cu} / \mathrm{Si}(001)$ ML capped by $20 \AA$ of $\mathrm{Cu}$. Measurements have shown that a 10.2 ML film of Ni has a perpendicular orientation of the magnetization vector. ${ }^{19}$

The values of the critical exponent $\beta$ of the magnetization $M(T)$ (Ref. 7) and Curie temperature depend upon the thickness of $\mathrm{Ni}(111)$ films on $\mathrm{W}(110)$ ( $\beta$ changes from 0.13 up to 0.30 between 5 and 7 layers). ${ }^{20}$ At a thickness near 7 layers a crossover from $2 \mathrm{D}$ to $3 \mathrm{D}$ Heisenberg magnetic state has 


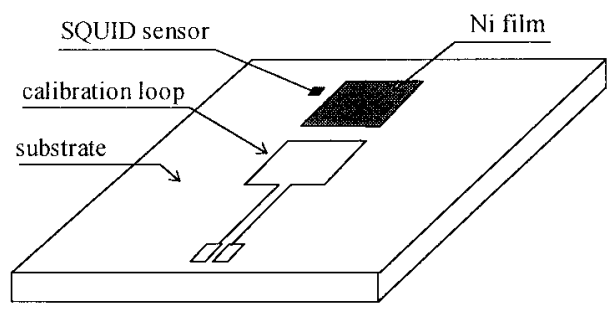

FIG. 1. Schematic of the experimental setup.

been detected in $\mathrm{Ni}$ film on $\mathrm{Cu}(001) .{ }^{21}$ The $\mathrm{Ni}$ films on $\mathrm{Cu}(100)$ and on $\mathrm{Cu}(111)$ exhibit a crossover from 3D Heisenberg to finite-size 2D behavior within the thickness range from about 7 to 12 layers. ${ }^{7}$ The Curie point of Ni/ $\mathrm{Cu}(001)$ monolayers varies from about 50 and up to about $460 \mathrm{~K}$ when the thickness of the films changes from about 1.5 monolayers up to $8 .^{22}$ The investigations of the finite-size scaling behavior of $T_{C}$ of the pseudomorphic Ni films (2.5-16 layers) have shown that the dependence of $T_{C}$ upon thickness can be extrapolated to $T_{C}=0$ for one $\mathrm{Ni}$ monolayer. ${ }^{21}$

Figure 1 shows a general arrangement of a SSM SQUID sensor, a sample film on (100) oriented $0.3 \mathrm{~mm}$ thick silicon substrate, and a thin film calibration loop deposited on the same silicon substrate. The shape of our samples was a square of $600 \times 600 \mu \mathrm{m}^{2}$. To map out a magnetic field distribution produced by the sample, the SSM scans over the sample area with a space separation of the order of $100 \mu \mathrm{m}$.

The thin film bicrystal HTS dc SQUID with outer dimensions $70 \times 70 \mu \mathrm{m}^{2}$ and inner hole $50 \times 50 \mu \mathrm{m}^{2}$ was used in this work. The measured effective pickup area, $A_{\text {eff }}$ of the SQUID was close to $3000 \mu \mathrm{m}^{2}$. The dc SQUID electronics was operated in a standard flux-locked mode; it detected the perpendicular to the SQUID plane magnetic field component, $B_{z}$, with a magnetic field sensitivity close to $100 \mathrm{pT} / \mathrm{Hz}^{1 / 2}$ at $1 \mathrm{~Hz}$ and $10 \mathrm{pT} / \mathrm{Hz}^{1 / 2}$ at $200 \mathrm{~Hz}$. The maximum signal bandwidth was about $5 \mathrm{kHz}$. The magnetizing field, oriented parallel to the sample plane, can be applied by the long solenoidal coil and the field, oriented perpendicular to the sample, by the 500-turn quasiflat wire coil. The SSM can operate properly in the fields up to about $70 \mathrm{~A} / \mathrm{m}$ for a field direction perpendicular to the SQUID and the sample plane and up to about $10^{4} \mathrm{~A} / \mathrm{m}$ for the parallel-oriented field.

The samples were fabricated by the following procedure: After the preparation of a liftoff resist mask on a monocrystalline (100) silicone wafer, it was placed in a vacuum deposition system Z-400 by Leybold. At a vacuum of 5 $\times 10^{-6}$ mbar the wafer was cleaned by sputtering at $10^{-2}$ mbar Ar, $900 \mathrm{~V}$ bias, two times for $30 \mathrm{~s}$ with a $30 \mathrm{~s}$ pause between. Subsequently, Ni was deposited by thermal

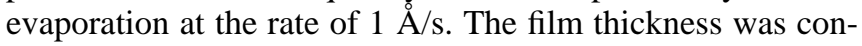
trolled by a Inficon quartz monitor. To preserve the thin $\mathrm{Ni}$ film from oxidation a $300 \AA$ layer of Au was deposited in situ by dc-magnetron sputtering at a $15 \AA /$ s rate. Finally, the resist mask was lifted off in acetone.

X-ray diffraction was performed on a thick Ni film (200 $\AA$ A) without Au covering using a the Rigaku diffractometer. Only the (111) reflection was found $\left(2 \Theta=44.32^{\circ}\right)$; the full width at half maximum was equal to $1.028^{\circ}$. The fact that only the (111) reflection was found reflects a textured struc- ture of the deposited Ni film with a strongly preferred (111) orientation. The lattice constant as calculated from the position of the $2 \Theta$ reflection was found to be $3.53 \AA$. The (111) orientation of the film seems reasonable; in this case the mismatch between the lattice constant of (100) oriented $\mathrm{Si}$ $(5.2 \AA)$ and $\mathrm{Ni}$ atoms in the film plane $(4.99 \AA)$ is minimal. We were unable to characterize thinner films in the same way due to the low intensity of the reflected $\mathrm{x}$ ray.

For the measurement procedure the SSM probe is immersed in a liquid nitrogen dewar surrounded by a shield of two layers of $\mu$ metal. The rotation of the SSM head around the vertical axis of $360^{\circ}$ gives the value of the horizontal component of the remnant magnetic field. Its maximum value was of the order of $2.5 \mu \mathrm{T}$. An ac current with an amplitude of about $300 \mu \mathrm{A}$ is fed to the calibration loop (see Fig. 1); its magnetic image allows convenient positioning of the sample within the scan area of the SSM (about 8 $\times 8 \mathrm{~mm}^{2}$ ). The measured magnitude of the magnetic field over the central part of the loop yields a value, $h$, of space separation between the sample and SQUID, which was varied in our experiments from 100 to $400 \mu \mathrm{m}$.

We studied the magnetic properties of Ni films with thicknesses between 8 and $200 \AA$ A. The films were covered by Au. The images were measured after cooling in a zero magnetic field from the initial temperature $T \approx 400 \mathrm{~K}$ down to liquid nitrogen temperature. After zero-field cooling, the $26 \AA$ film produced a weak magnetic image with an in-plane magnetization. When a magnetizing field of the order of $4000 \mathrm{~A} / \mathrm{m}$ was applied parallel to the plane of the film a clear magnetic image, shown in Fig. 2(a), had appeared. One can see the sharp maximum and minimum of the measured $B_{z}$ component located near the opposite edges of the sample film, marked in Fig. 2(a) by a dashed line. The symmetry of the image (equal absolute values of the positive and negative values) is caused by the magnetization of the film. The existence of only two peaks in the magnetic image of the entire area can be interpreted as the signature of a monodomain structure. It is important to note that the space resolution of SSM is at least one order of magnitude better than the film sizes, and that the signal-to-noise ratio is better than a factor of 100 .

The value of the measured magnetic flux density component $B_{z}(x, y)$ with respect to the background signal $B_{z}(0,0)$, recorded far from the film, was obtained from the SQUID output voltage $\Delta V_{\text {out }}$, the SQUID's feedback constant $\Delta V_{\text {out }} / \Phi_{0}$ (here $\Phi_{0}$ is a flux quantum) and from the previously measured effective area $A_{\text {eff }}$. To find the value of the film magnetic moment $m_{f}$, one can use Fig. 2(b) and the classic formulas of magnetism. The value of the magnetic field $d \mathbf{B}$ at the measuring point $(X=u, Y=v, Z=h)$ produced by the elementary magnetic moment $\mathbf{m}$ (a film magnetic moment per unit area) with coordinates $(X=x, Y=y$, $Z=0)$ is

$$
d \mathbf{B}=\frac{\mu_{0}}{4 \pi} \times \frac{(3 \mathbf{n} \cdot \mathbf{m}) \mathbf{n}-\mathbf{m}}{|\mathbf{R}|^{3}},
$$

where $\mathbf{n}$ is a unit vector along $\mathbf{R}$. Assuming that $\mathbf{m}$ has only an $m_{x} \equiv m$ component [in-plane magnetization of the film in accordance with Fig. 2(a)] the total value of $B_{z}(u, v, h)$ is 

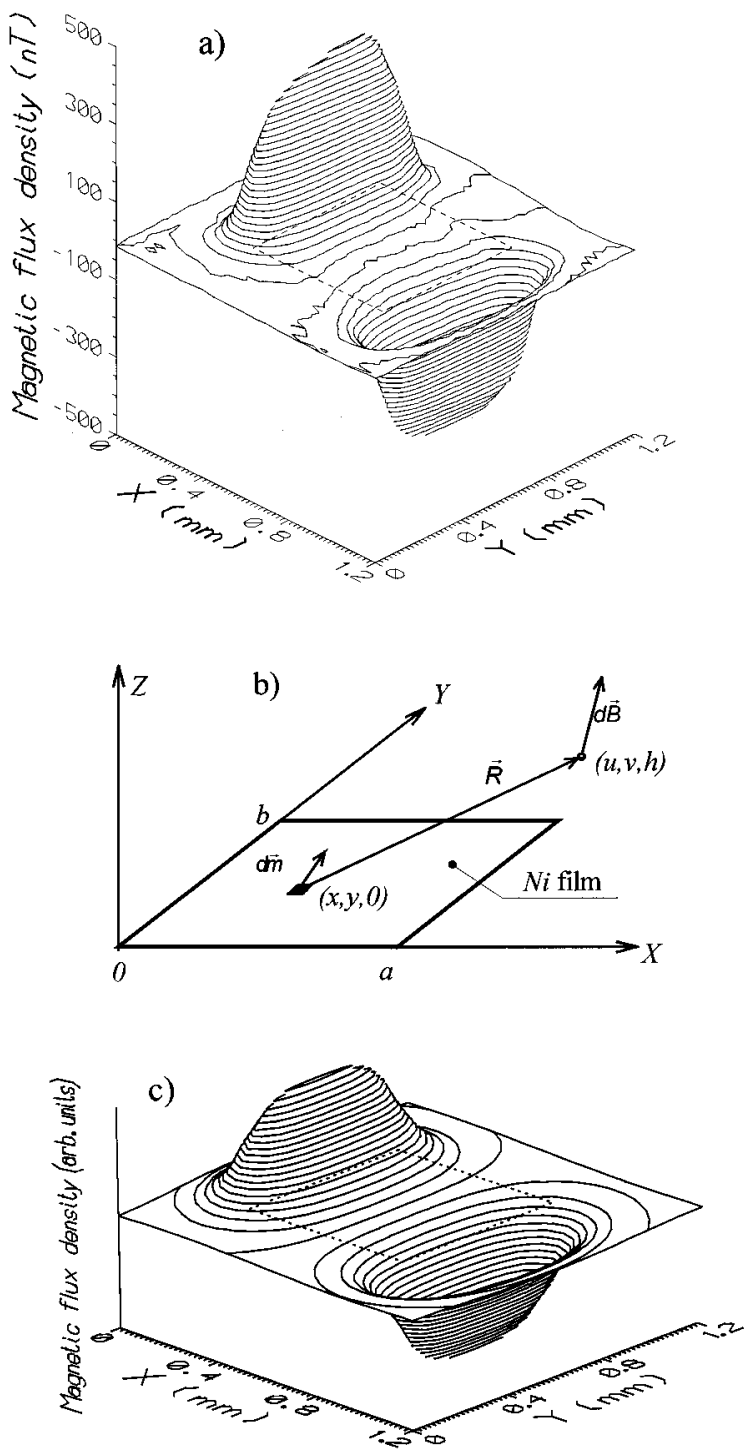

FIG. 2. (a) Magnetic image of the $0.6 \times 0.6 \mu \mathrm{m}^{2} 26 \AA$ thick Ni film in magnetizing field equal to $4000 \mathrm{~A} / \mathrm{m}$, applied parallel to axis $Y$. (b) Schematic used in calculations. (c) Example of the reconstructed image.

$$
B_{z}^{\mathrm{tot}}(u, v, H)=\frac{\mu_{0}}{4 \pi} \times 3 h m \int_{0}^{a} \int_{0}^{b} \frac{u-x}{|R|^{5}} d x d y .
$$

Equation (2) leaves one parameter, $m$, for fitting. An example of a calculated image is shown in Fig. 2(c). By fitting the calculated to the measured image one can find the absolute value of the total magnetic moment. The sample volume magnetization of $26 \AA$ thick film vs applied parallel field is shown in Fig. 3. This hysteresis curve was obtained in successive measurements of the maximum value of $B_{z}$ when the applied field was changed from plus $4000 \mathrm{~A} / \mathrm{m}$ to minus $4000 \mathrm{~A} / \mathrm{m}$ and back. Our measurements indicate that the value of saturation magnetization in the film is $0.17 \mathrm{MA} / \mathrm{m}$ and reached at the field strength of about $2500 \mathrm{~A} / \mathrm{m}$. This value of saturation magnetization is approximately three times less than for bulk Ni.

The perpendicular field with the maximum magnitude 70 $\mathrm{A} / \mathrm{m}$ was produced in $26 \AA$ film signals at $Z_{0}$ altitude below

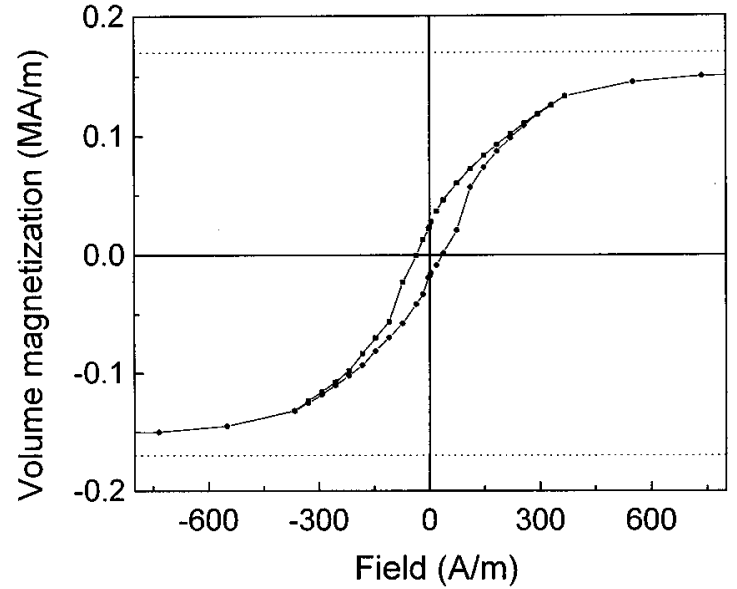

FIG. 3. Hysteresis loop of the $26 \AA \mathrm{Ni}$ thin film sample in parallel applied field.

the SQUID noise level and no images were obtained under such field orientation. The hysteresis loop (see Fig. 3) indicates the presence of ferromagnetic ordering in the sample, with a Curie temperature higher than $77 \mathrm{~K}$. A coercivity magnitude close to $35 \mathrm{~A} / \mathrm{m}$ and remnant magnetic moment of about $0.028 \mathrm{MA} / \mathrm{m}$ were observed.

Attempts to take images of the samples with the thickness 15 and $8 \AA$ in parallel and perpendicular fields with about $100 \mu \mathrm{m}$ space separation from the SQUID indicate that the value of magnetization was below the sensitivity threshold. In the thickness range 15-26 $\AA$ the value of the volume magnetization thus decreases by more than two orders of magnitudes (see Fig. 4).

Contrary to $26 \AA$ film, the Ni films with thicknesses 43 and $84 \AA$ (also coated by $\mathrm{Au}$ ) after cooling to $77 \mathrm{~K}$ in the zero field have a clearly recorded pair of the symmetric positive and negative peaks in images, placed in opposite corners of the square of the film. Such images corresponds to singledomain magnetic structures with an in-plane easy-axis. When the parallel magnetizing field with a magnitude of the order of $4000 \mathrm{~A} / \mathrm{m}$ was applied, the magnetic moment of the film was rotated to the field direction and the peaks shifted to the midline position, as is shown in Fig. 2(a). Similar results was obtained for the $200 \AA$ film without Au coating.

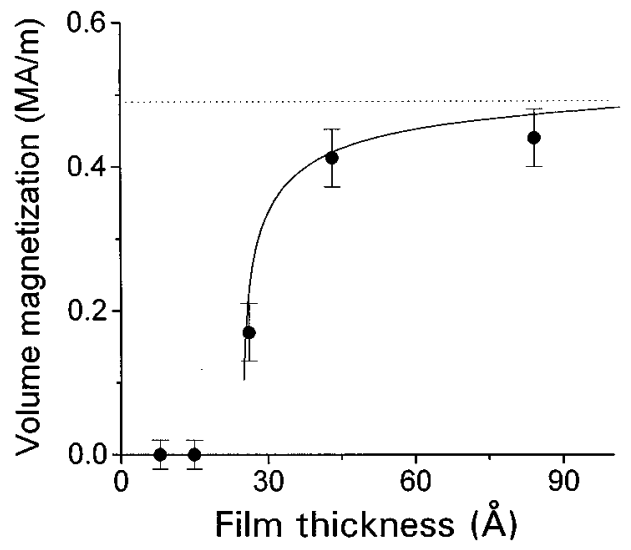

FIG. 4. Thickness dependence of the saturation magnetization in $\mathrm{Au} / \mathrm{Ni} / \mathrm{Si}(100)$ films. 
The hysteresis loops of films with thickness greater than $26 \AA$ can be recorded in an applied magnetic field with magnitude up to $4000 \mathrm{~A} / \mathrm{m}$ when the field is parallel both to the sample and SQUID plane. In the case of a perpendicular magnetizing field, SQUID's with submicron junctions must be used. ${ }^{23}$ The space and magnetization resolutions of this method at present are close to $100 \mu \mathrm{m}$ and $10^{-15} \mathrm{~A} \mathrm{~m}^{2} / \mathrm{Hz}^{1 / 2}$, respectively. These parameters can be improved by decreasing the space separation between the SQUID and sample.

A distinct change of magnetic behavior of the Ni layers thinner than 10-20 $\mathrm{A}$ from the bulk Ni has previously been demonstrated in the Ni/Pt multilayers. ${ }^{24}$ An almost constant value of the saturation magnetization $M_{0}=1.78 \mathrm{MA} / \mathrm{m}$ (equal to that for bulk $\mathrm{Fe}$ ) of the polycrystalline $\mathrm{Fe}$ films for thickness from 1000 down to $30 \AA$ has been observed. ${ }^{5}$

The value of the coercive field in the thickness range 30-50 ̊ was considerably smaller than for films that are much thicker. Theoretical consideration ${ }^{25,26}$ shows that the 2D Heisenberg layers may have a reorientation temperature point $T_{R}$, where magnetization of the monolayer rotates from out-of-plane to in-plane and the planar magnetic structure is valid above the phase transition temperature $T>T_{R}$.

Our experiments demonstrated that in one-domain $\mathrm{Au} / \mathrm{Ni} /$ $\mathrm{Si}(100)$ films, the in-plane magnetization at $T=77 \mathrm{~K}$ in zero field appears only for thicknesses greater than $26 \AA$ (first experimental point $43 \AA$ ). This behavior may be a result of the films being expanded by the $\mathrm{Si}$ substrate and consequently have an in-plane easy direction, and/or because at the temperature $T=77 \mathrm{~K}$ the films are in a temperature range between the Curie point and a reorientation temperature where in-plane magnetization is permissible.

The values of the saturation magnetization in the 43 and
$84 \AA$ films are close to the bulk value (see Fig. 4), which is in accordance with theoretical predictions. ${ }^{14}$ The decreased magnetization value observed for the $26 \AA$ film is most likely caused by $T=77 \mathrm{~K}$ being close to the Curie point for this film thickness. Superparamagnetic behavior for Fe films at a thickness below $30 \AA$ has been detected, ${ }^{5}$ and it was suggested that this is a typical magnetic property for film which had formed islands during growth.

The transition to in-plane ferromagnetic behavior of the magnetic structure at an average island radius $a>30 \AA$ (at about $13 \mathrm{ML}$ of $\mathrm{Fe}$ ) has been observed. ${ }^{27}$ For the Fe clusters with a radius of $44 \AA$ the local order parameter has a nonzero value up to $580 \mathrm{~K} .{ }^{27}$ Thus a change of magnetic behavior in our Ni film in the thickness range 15-26 A may also be due to cluster formation. The domain pattern of our thick films had a small sensitivity to the field, whether applied parallel or perpendicular to the film plane (in our experiments up to 4000 and $70 \mathrm{~A} / \mathrm{m}$, respectively).

No magnetic moment was detected for the 8 and $15 \AA \mathrm{Ni}$ films with current level of sensitivity. Most likely their Curie point is below $77 \mathrm{~K}$, although the mechanisms discussed in Ref. 28 may also play a role. In conclusion, the developed SQUID method is able to take images of magnetic structures of Ni films to determine the magnetization of ultrathin magnetic films, which allows us to determine quantitatively the local magnetization magnitude.

The work was supported in part by Russian Fund of Basic Research, Grants Nos. 96-02-19250, 96-02-18127a, and INTAS Grant No. 93-2777- ext. We would like to thank S. I. Krasnosvobodtsev, L. V. Matveets, A. B. Pavolotsky, S. N. Polyakov, N. N. Ukhansky, and I. I. Vengrus for help at different stages of this work.
${ }^{1}$ N. D. Mermin and H. Wagner, Phys. Rev. Lett. 17, 1133 (1966).

${ }^{2}$ L. Onsager, Phys. Rev. 65, 117 (1944).

${ }^{3}$ M. Sakurai, N. Imamura, K. Hirano, and T. Shinjo, J. Magn. Magn. Mater. 147, 16 (1995).

${ }^{4}$ R. Bergholz and U. Gragmann, J. Magn. Magn. Mater. 45, 389 (1984).

${ }^{5}$ M. Weber, R. Koch, and K. H. Rieder, Phys. Rev. Lett. 73, 1166 (1994).

${ }^{6}$ G. Bochi, H. J. Hug, D. I. Paul, B. Stiefel, A. Moser, I. Parashikov, H. J. Güntherodt, and R. C. O'Handley, Phys. Rev. Lett. 75, 1839 (1995).

${ }^{7}$ F. Huang, M. T. Kief, G. J. Mankey, and R. F. Willis, Phys. Rev. B 49, 3962 (1994)

${ }^{8}$ G. Bochi, C. A. Ballentine, H. E. Inglefield, C. V. Thompson, R. C. O'Handley, H. J. Hug, B. Stiefel, A. Moser, and H. J. Güntherodt, Phys. Rev. B 52, 7311 (1995).

${ }^{9}$ C. F. Majkrzak, J. Kwo, M. Hong, Y. Yafet, D. Gibbs, C. L. Chien, and J. Bohr, Adv. Phys. 40, 99 (1991).

${ }^{10}$ G. M. Watson, D. Gibbs, G. H. Lander, B. D. Gaulin, L. E. Berman, H. Matzke, and W. Ellis, Phys. Rev. Lett. 77, 751 (1996).

${ }^{11}$ G. M. Watson, D. Gibbs, G. H. Lander, B. D. Gaulin, L. E. Berman, H. Matzke, and W. Ellis, Phys. Rev. Lett. 77, 751 (1996)
${ }^{12}$ L. N. Vu, M. S. Wistrom, and D. J. Van Harlingen, Appl. Phys. Lett. 63, 1693 (1993).

${ }^{13}$ J. R. Kirtley, M. B. Ketchen, K. G. Stawaisz, J. Z. Sun, W. J. Gallagher, S. H. Blanton, and S. J. Wind, Appl. Phys. Lett. 66, 1138 (1995).

${ }^{14}$ V. L. Moruzzi, P. M. Marcus, K. Schwarz, and P. Mohn, Phys. Rev. B 34, 1784 (1986).

${ }^{15}$ J. I. Lee, Soon C. Hong, A. J. Freeman, and C. L. Fu, Phys. Rev. B 47, 810 (1993).

${ }^{16}$ J. Voigt, X. L. Ding, R. Fink, G. Krausch, B. Luckscheiter, R. Platzer, U. Wührmann, and G. Schatz, Phys. Rev. Lett. 66, 3199 (1991).

${ }^{17}$ J. Vogel, G. Panaccione, and M. Sacchi, Phys. Rev. B 50, 7157 (1994).

${ }^{18}$ B. Schulz and K. Baberschke, Phys. Rev. B 50, 13467 (1994).

${ }^{19}$ J. Shen, J. Giergiel, and J. Kirschner, Phys. Rev. B 52, 8454 (1995).

${ }^{20}$ Yi. Li and K. Baberschke, Phys. Rev. Lett. 68, 1208 (1992).

${ }^{21}$ F. Huang, G. J. Mankey, M. T. Kief, and R. F. Willis, J. Appl. Phys. 73, 6760 (1993).

${ }^{22}$ A. Aspelmeier, M. Tischer, M. Farle, M. Russo, K. Baberschke, and D. Arvanitis, J. Magn. Magn. Mater. 146, 256 (1995).

${ }^{23}$ M. Vaupel, G. Ockenfuss, and R. Wordenber, Appl. Phys. Lett. 68, 3623 (1996). 
${ }^{24}$ R. Krishnan, H. Lassri, Shiva Prasad, M. Porte, and M. Tessier, J. Appl. Phys. 73, 6433 (1993); R. Krishnan, H. Lassri, M. Seddat, M. Porte, and M. Tessier, Appl. Phys. Lett. 64, 2312 (1994).

${ }^{25}$ D. Pescia and V. L. Pokrovsky, Phys. Rev. Lett. 65, 2599 (1990).

${ }^{26}$ Ar. Abanov, V. Kalatsky, V. L. Pokrovsky, and W. M. Saslow,
Phys. Rev. B 51, 1023 (1995).

${ }^{27}$ M. R. Schenfein, K. E. Schmidt, K. R. Heim, and G. G. Hembree, Phys. Rev. Lett. 76, 1541 (1996).

${ }^{28}$ C. P. Flynn and M. B. Salamon, in Handbook of the Physics and Chemistry of Rare Earth, edited by K. A. Gschneidner, Jr. and L. Eyring (North-Holland, Amsterdam, 1996), Vol. 22, p. 147. 